First Peoples Child \& Family Review

An Interdisciplinary Journal Honouring the Voices, Perspectives, and Knowledges of First Peoples through Research, Critical Analyses, Stories, Standpoints and Media Reviews

\title{
Diagnosis as a Naming Ceremony: Caution Warranted in Use of the DSM-IV with Canadian Aboriginal Peoples
}

\section{Danika Overmars}

Volume 5, Number 1, 2010

URI: https://id.erudit.org/iderudit/1069064ar

DOI: https://doi.org/10.7202/1069064ar

See table of contents

Publisher(s)

First Nations Child and Family Caring Society of Canada

ISSN

1708-489X (print)

2293-6610 (digital)

Explore this journal

Cite this article

Overmars, D. (2010). Diagnosis as a Naming Ceremony: Caution Warranted in

Use of the DSM-IV with Canadian Aboriginal Peoples. First Peoples Child \&

Family Review, 5(1), 78-85. https://doi.org/10.7202/1069064ar

\section{Article abstract}

The Diagnostic and Statistical Manual of Mental Disorders, 4th is the primary tool for diagnosis of mental disorders used in the field of mental health. Despite the widespread use of the DSM-IV there are limits to its applications, particularly with Canadian Aboriginal people. This paper draws parallels between the process of diagnosis and an Aboriginal naming ceremony used by the Coast-Salish people in British Columbia. Caution is suggested when applying Western based diagnoses to Aboriginal people due to the lack of cultural relevance and recommendations are made for appropriate use. Edition (DSM-IV; APA, 1994)
This document is protected by copyright law. Use of the services of Erudit (including reproduction) is subject to its terms and conditions, which can be viewed online.

https://apropos.erudit.org/en/users/policy-on-use/ 


\title{
Diagnosis as a Naming Ceremony: Caution Warranted in Use of the DSM-IV with Canadian Aboriginal Peoples
}

\author{
Danika Overmars ${ }^{a}$
}

a Master of Arts Student at Ontario Institute for Studies in Education at the University of Toronto, Department of Adult Education and Counselling Psychology.

"What's in a name? That which we call a rose by any other name would smell as sweet.

\section{Shakespeare; Romeo and Juliet (II, ii, 1-2)}

Although the truth of Shakespeare's words apply in many respects, this paper contests that in the field of mental health a name, or diagnosis, can have a profound effect on an individual, particularly those of Canadian Aboriginal backgrounds in whose cultures naming is an important ceremony. In mental health services the Diagnostic and Statistical Manual of Mental Disorders (4th Edition) Text Revision (DSM-IV-TR) that is published by the American Psychiatric Association (APA; $2000)$ is where the criteria, names and classifications for diagnoses are found. This paper refers to the DSM-IV(APA; 1994), rather than the DSM-IV-TR, because the majority of available literature addresses the DSM-IV. This paper explores the use of the DSM-IV with Aboriginal populations and parallels the process of diagnosis with that of naming ceremonies. The paper concludes with implications for mental health services.

Mental health has been defined as the absence of disease (Stewart, 2007) rather than focussing on the wellbeing of the individual. However, the definition has evolved and a recent rendition from Health Canada (2000; as cited in Stewart, 2007, p. 54) states that "mental health is the capacity of the individual, the group and the environment to interact with one another in ways

Questions or correspondence concerning this article may be addressed to:

dovermars@oise.utoronto.ca

\section{Abstract}

The Diagnostic and Statistical Manual of Mental Disorders, 4th Edition (DSM-IV; APA, 1994) is the primary tool for diagnosis of mental disorders used in the field of mental health. Despite the widespread use of the DSM-IV there are limits to its applications, particularly with Canadian Aboriginal people. This paper draws parallels between the process of diagnosis and an Aboriginal naming ceremony used by the Coast-Salish people in British Columbia. Caution is suggested when applying Western based diagnoses to Aboriginal people due to the lack of cultural relevance and recommendations are made for appropriate use.

that promote subjective well-being, the optimal development and use of mental abilities (cognitive, affective and relational), the achievement of individual and collective goals consistent with justice and the attainment and preservation of conditions of fundamental equality." The most recent definition goes well beyond the absence of disease and talks about mental health as it might be seen from a holistic perspective. Yet, the definition does not refer to connections with physical health or spirituality; which, from an Indigenous perspective neglects to acknowledge the interconnectedness of the individual as the cohesive whole described in the Medicine Wheel teachings.

The Medicine Wheel teachings depict the person as composed of four facets: the spirit, the emotions, the intellect and the physical (Bopp \& Bopp, 2001). Though these components are distinct from one another they are inextricably interconnected; one component cannot be affected without affecting the others. For example, consider a person who is depressed; researchers have noted that depressed patients exhibit a change in their thought patterns (Beck, 1991) which reflects the impact on intellect. The impact on the physical body is seen in changes in sleep patterns, feelings of fatigue and pain (Greden, 2003; Leahy \& Holland, 2000). Emotions are perhaps the most prominently affected by depression as 
the person often feels overwhelmed by feelings of sadness, inadequacy and hopelessness (Leahy \& Holland, 2000). Duran (2006) suggests that the spirit is also affected as the person is visited by the spirit of sadness. As such, one can see that when one part of the person is afflicted by an illness or injury there is an impact on every aspect of the person. Consequently, it seems reasonable that healing should address all aspects of the individual rather than focussing on only one component.

The term Indigenous paradigm refers to the shared cultural attitudes and beliefs of Indigenous people while the term Western paradigm refers to dominant attitudes and beliefs that are informed by and based on Western European philosophies and practices (Stewart, 2007). According to Vicary and Bishop (2005), Aboriginal conceptions of health are holistic and include both a cultural and spiritual aspect that is often ignored in the Western paradigm. One of the most conspicuous differences between Western and Indigenous approaches to health, and to the world in general, is the Western model's penchant for compartmentalization while Indigenous models focus on cohesiveness and interconnectedness (Steinhauer, 2002). The entire Western health care system is set up in this way; there is a psychiatrist to help with difficulties of the mind, a cardiologist to help with heart problems, a gastroenterologist to help with problems of the digestive system, and the list goes on. This is not to say there is no role for specialized knowledge or specialists yet when they focus on one aspect of the person to the exclusion of the rest of the person it is hard to reconcile the process with an Indigenous paradigm.

There are significant and numerous differences between Western and Indigenous paradigms and these differences are thought to contribute to the underuse of services by Aboriginal people in Canada, despite the high levels of mental health problems documented (Kirmayer, Brass \& Tait, 2000; Kirmayer, Simpson \& Cargo, 2003; Stewart, 2008). The problems include, but are not limited to: alcoholism, suicide, depression, anxiety, substance abuse, post traumatic stress disorder, and conduct disorder. Furthermore, as Kirmayer et al. (2000) point out, the prevalence of these problems is underrated because of the fact that Aboriginal people are not using the mental health services that are currently provided. Although the incongruence of Western and Indigenous worldviews contributes to the underuse of services, several authors (Duran, 2006; Kirmayer et al., 2003; Stewart, 2008) also suggest that the legacy of colonization and historical trauma are correlated with decreased service use because current services are not culturally relevant. Stewart discusses how the majority of mental health services available to Aboriginal people are based on Western conceptualizations of health and healing that may not value the worldview of
Aboriginal clients. Stewart goes on to suggest that for healing to occur a cultural approach to mental health and healing needs to be developed. Finally, Stewart concludes that for Aboriginal clients, healing from colonialism continues to be a significant mental health concern.

Colonialism, as outlined in the article by Schissel and Wotherspoon (2003), is an ongoing process whereby dominant cultural groups subordinate or regulate Aboriginal populations. As a result of colonization, Aboriginal people in Canada have suffered innumerable injustices; one of the most prominent and destructive examples of colonization is the residential school system (Schissel \& Wotherspoon, 2003). The residential school system has not only influenced those who attended the schools but their families, friends, communities, and the following generations. A term that has been used to describe the continued deleterious influence of residential school is historical trauma. Historical trauma is "the legacy of numerous traumatic events a community experiences over generations and encompasses the psychological and social responses to such events" (Evans-Campbell, 2008, p. 320). Research suggests that clinicians in the field of mental health need to acknowledge and understand the impact of historical trauma, especially residential school, on all Aboriginal people in order to work with them in a culturally sensitive way (Duran, 2006; Duran, Firehammer, \& Gonzales, 2008; Kirmayer et al., 2003; Stewart, 2008). Neglecting to acknowledge the impact of historical trauma has been described as psychological oppression (Duran, 2006) and continued colonization in the field of mental health (McIntyre, 1996; Stewart, 2008).

Stating that there is psychological oppression or colonization within the mental health field may seem an extreme statement but when taking a closer look it seems less radical. A mental health practitioner, although well-intentioned, using therapeutic interventions that are Western-based and not responsive to Indigenous values or worldviews is, in effect, promoting mainstream values and encouraging conformity to these values (Duran, 2008; McIntyre, 1996; Stewart, 2008). As a result, the continued colonization is more often due to a lack of cultural sensitivity, knowledge and understanding, than as a result of malicious intent. However, many interventions and clinical tools, such as the DSM-IV-TR, neglect to thoroughly address the cultural component and, as such, this problem is ingrained into the current systems and process.

\section{Diagnostic and Statistical Manual of Mental Disorders}

The Diagnostic and Statistical Manual of Mental Disorders was first published by the American Psychiatric Association in 1952 (Grob, 1991) and the most recent 
revision the Diagnostic and Statistical Manual of Mental Disorders (Fourth Edition) Text Revision became available in 2000. The text revision did not change any of the diagnostic criteria or categories of the DSM-IV; however, supplemental information was added to many of the current categories (APA, 2000; Dziegielewski, 2002). Nonetheless, the majority of the available literature refers to the DSM-IV rather than the DSM-IV-TR. The APA (2009) describes the DSM-IV as "the standard classification of mental disorders used by mental health professionals... for use across clinical settings (inpatient, outpatient, partial hospital, consultation-liaison, clinic, private practice, and primary care), with community populations." The DSM-IV is what one might call a gold-standard in the field of mental health when it comes to the diagnosis of mental disorders. According to Dziegielewski (2002) psychiatrists, psychologists, psychiatric nurses, social workers, and other mental health professionals all utilize the DSM-IV in clinical practice.

The DSM-IV consists of a list of disorders each of which has a set of symptom criteria and descriptive text (APA, 2009). The DSM-IV typically requires five or more symptoms to persist for two or more weeks in order for a diagnosis to occur (Leahy \& Holland, 2000). The DSM-IV (1994) has five clinical axis which are: Axis I, clinical disorders, including major mental disorders, as well as developmental and learning disorders; Axis II, underlying pervasive or personality conditions, as well as mental retardation; Axis III, Acute medical conditions and physical disorders; Axis IV, psychosocial and environmental factors contributing to the disorder; Axis V, Global Assessment of Functioning. The DSMIV-TR contains codes for all of these disorders which are used in the medical insurance billing process in the United States and are also widely used as diagnoses for medical insurance purposes in Canada (Kirmayer, 1998).

The DSM-IV attempts to include culture in four places within the manual (Kirmayer, 1998). The first is within the introduction where the manual makes reference to the importance of culture. The second place is in the text description that accompanies the disorders. The third place is in the "Outline for Cultural Formulation" in Appendix I and the final component is the list of culture-bound syndromes, also included in the appendix. Good (1996) describes the list of culture-bound syndromes as a "glossary of cultural terms" (p. 128).

The "Outline for Cultural Formulation" includes five processes, or steps, in which the clinician is to engage (Manson, 2000). The first step is for the clinician to inquire about the client's cultural identity, asking questions about language preference and use, religious identity and cultural reference group. In working with Aboriginal people this is very important given the heterogeneity of the communities that are given the title of Aboriginal (Kirmayer et al., 2000). In Canada, Aboriginal people are the only group whose identity is legally defined (Gibbins, 1997 as cited in Offet-Gartner, 2008); the definition is provided in the Indian Act of 1876. In Canada there are three main group of Aboriginal people which are First Nations, Métis, and Inuit (Schissel \& Wotherspoon, 2003).

Next, the clinician's role is to discuss and explore cultural explanations of the illness. According to Kirmayer's (2004) report in the British Medical Journal, 20-40\% of adults use various forms of complementary and alternative medicine. As such, it is important to talk to clients about their pre-existing notions of what is afflicting them and what they may have learned from traditional healers within their community. The third step is to consider the relevance of cultural factors within the psychosocial environment of the client. In working with Aboriginal people this is a place where the clinician may consider the impacts of historical trauma because it significantly impacts the psychosocial environment in a detrimental manner (Evans-Campbell, 2008). This is also an opportunity for the clinician to explore cultural and community support systems, in addition to assessing the level of functioning as well as the disability. An examination of the relationship dynamics between the clinician and client is the fourth step in the Outline for Cultural Formulation. This entails examining the differences in status, both culturally and socially; it involves the clinician taking a careful look at the power imbalances present in the system and considering the impacts it may have on interactions with the client. Finally, the clinician is asked to synthesize all the previously gathered information to render a cultural assessment to go alongside the diagnosis and discuss the implications for treatment and care. For a clinical demonstration of the use of the "Outline for Cultural Formulation" see Novins and colleagues (1997).

\section{Strengths and Limitations of the DSM-IV-TR}

The clinical utility of the DSM-IV-TR is displayed by the prevalence of its use in both Canada and the US (Dziegielewski, 2002). Mental health practitioners are able to use it in a formulaic manner to arrive at a diagnosis for clients; if a client displays symptoms $\mathrm{A}$ through $\mathrm{C}$ for the duration of $\mathrm{X}$ weeks then she/he has disorder 1. The DSM-IV-TR is designed to remove the subjectivity from the diagnostic process in order to provide consistent diagnoses (Dziegielewski, 2002).

The inclusion of codes for disorders in the DSM-IV simplifies and helps to standardize the reporting procedures and process, especially in the US where health care is privatized (Dziegielewski, 2002). This allows health care practitioners to 
reduce the time spent filing paper work and the streamlining of the diagnostic process is a significant benefit of the DSM-IV. Rather than a health care practitioner spending time developing an individual diagnosis for each client, they are able to asses a client's history and current symptoms to determine whether a client meets the criteria for a diagnosis of a particular disorder. Once a diagnosis is made, the clinician is able to determine the appropriate treatment. For example, once a clinician determines that a client meets the criteria for depression, he or she may investigate the options for taking antidepressant medication and/or undergoing cognitive-behavioural therapy (Leahy \& Holland, 2000). In this way the DSM-IV saves time and promotes the development of effective treatments for the symptom clusters that are known as disorders. Furthermore, the DSM-IV enables uniform and standardized diagnosis (Dziegielewski, 2002).

The DSM-IV is beneficial because it provides a systematic description of disorders and does capture the features of disorders that are common across cultures (Csordas, Storck, \& Strauss, 2008). As Csordas et al. (2008) point out, the DSM-IV is the best categorization method available at this point and as such should be used. Nonetheless, with the ability of the DSMIV to capture common themes across cultures practitioners must also be cognizant of the coinciding limitation: the categories and classifications used are a product of Western cultures and their generalizability must be critically examined before being applied to people of diverse cultural backgrounds, such as individuals from Aboriginal cultures.

The DSM-IV is based in a Western-scientific positivist model (Kirmayer, 1998). Although this may be a strength when working with people in Western cultures, it is a limitation when working with people of Aboriginal ancestry because the values the DSM-IV is based on are incongruent with Aboriginal values and worldviews. For example, the categories in the DSM-IV are based on scientific and empirical knowledge where there is only one truth, whereas in Aboriginal cultures there are multiple truths depending upon who you consult with (Steinhauer, 2002). Moreover, knowledge can be derived from multiple sources which include traditional teachings, empirical observations, and revelations (Castellano, 1999). Kirmayer (1998) suggests any changes made to the DSM-IV must be supported by large-scale epidemiology and clinical validation studies, which limit the research that can be included simply because the costs of the research are astronomical. As such, the DSM-IV only recognizes the Western ways of knowing and ignores Indigenous Ways of knowing, particularly those pertaining to the spiritual aspect of people.

Manson (2000) discusses the idea that the DSM-IV needs to incorporate a more holistic conception of the individual.
The DSM-IV considers the mental, emotional and physical aspects of the person yet ignores the spiritual aspect of the person. Spirituality is a central component to Aboriginal worldviews and values (Steinhauer, 2002) and to disregard it is to disregard an integral component of the individual. A study by Csordas and colleagues (2008) attempts to bridge the gap between the clinical diagnoses provided by the DSM-IV and the understanding of illness provided by traditional healers in an Aboriginal community. The authors suggest that attention needs to be paid to connections between spirituality and mental health because the DSM-IV does not address them. However, this lack of acknowledgement for spirituality may be a reflection of how healing practices and spirituality are currently conceptualized in Western cultures and in the Western medical system which produces the DSM-IV.

The DSM-IV is also limited as it only includes large scale research and excludes research findings potentially relevant to a smaller proportion of the population (Kirmayer, 1998). The DSM-IV contains a category for posttraumatic stress disorder (PTSD), yet does not contain a category for historical or intergenerational trauma; authors describing historical trauma argue that historical trauma is distinct from posttraumatic stress disorder and has a different impact on people (EvansCampbell, 2008; Whitbeck, Adams, Hoyt \& Chen, 2004). According to Evans-Campbell, PTSD does not address "the additive effects of multiple traumatic events occurring over generations" (p. 317) nor does it explore how trauma can be transmitted within a community or down through the generations. Evans-Cambell, among others, would suggest that disregarding the impact of historical trauma on mental health is doing a great disservice not only to Aboriginal peoples but to other cultural groups which suffer from intergenerational trauma, such as holocaust survivors and newcomers from war zones.

As opposed to earlier versions of the manual, the DSMIV made a concerted effort to include culture (Good, 1996). However, the DSM-IV's "Outline for Cultural Formulation" is included as an Appendix at the back of the manual, rather than at the front following the introduction where Kirmayer (1998) suggests that it would indicate the importance of placing diagnosis in a cultural context. In this way, culture is treated as an add-on to mainstream practices rather than being valued as a significant influence on the mental health of the individual. This de-valuing the influence of culture is a continued form of oppression and colonization (Duran, 2006). Strengths and limitations notwithstanding, the DSM-IV-TR is a widely used clinical diagnosis tool that has the potential to be immensely helpful if used in a culturally appropriate manner. 


\section{Diagnosis as a Naming Ceremony}

\section{Diagnosis as Naming}

There are a number of parallels between the process of being given a diagnosis and a naming ceremony in Indigenous cultures. These parallels should give mental health care practitioners pause when they use diagnostic tools and labels, such as those evidenced in the DSM-IV, with Indigenous peoples. Given that naming ceremonies are traditional practices there is not an abundance of literature on the subject. However, based on the description provided by Thom (2003) in his presentation on Coast-Salish First Nations communities, analogies can be made between the processes and procedures, how the name/diagnosis is meant to describe the person, and how the name is given to the person by someone of significance.

A naming ceremony, among the Coast-Salish First Nations communities, occurs as formal ceremony in which certain prerequisite performances, such as sxwayxwey mask dance, are completed before the name is given (Thom, 2003). These prerequisite performances can be likened to the interview and assessment process that occurs before a diagnosis is given. In a naming ceremony an Elder or family spokesperson then announces the name and calls on the older generations present to bear witness (Thom, 2003). This is analogous to the process whereby a clinician formulates a diagnosis and then consults with other clinicians (and possibly reference manuals such as the DSM-IV-TR) to confirm and validate the diagnosis. It is interesting to note that the names given accord the bearer certain privileges, much the same way a diagnosis can secure a client certain social services and accommodations (Dziegielewski, 2002). As Dziegielewski explains, certain diagnoses qualify clients for more agency services and without a diagnosis a client may not qualify for services at all. Dziegielewski also notes that some clinicians are inclined to give a more severe diagnosis to allow the client to qualify for increased services while other clinicians are inclined to provide the least severe diagnosis to avoid stigmatizing and labelling clients.

The stigma that results from labelling is a significant concern in the field of mental health; the level of concern is reflected in the fact that there are entire theories, such as Labelling theory (Markowitz, 1998), to address the impact of labelling. Labelling theory, according to Markowitz, contends that individuals who are labelled with a diagnosis expect to be discriminated against and devalued as a result of stereotypic beliefs about people who are mentally ill. As a result, these beliefs act as a self-fulfilling prophecy and can impact psychological well being and life satisfaction. Van Den Tillaart, Kurtz and Cash (2009) suggest that diagnosis not only stigmatizes people, but also serves to marginalize them. Furthermore, this is especially troubling for those clients who are considered to be part of a marginalized population before they receive a diagnostic label.
One of the strongest parallels between a naming ceremony and a diagnosis is the fact that the name is given to an individual by someone who occupies a position of respect and power. In the Coast-Salish tradition an Elder or family member gives the name (Thom, 2003). In the mental health field a diagnosis is given by a registered mental health professional such as a psychologist or psychiatrist. Both parties are assumed to have the knowledge and wisdom to gives names appropriately and with careful consideration of the characteristics of the person who is to receive the name.

Duran (2006) also provides insight into the parallels between diagnosis and naming ceremonies. Duran discusses how naming ceremonies are meant to provide people with spiritual names that are reflective of their individual identities. These naming ceremonies can have a transformative effect on people. Diagnosis can act in much the same way, when a person goes to a mental health professional seeking help and advice, they are often looking for a name for whatever is afflicting them. Mental health practitioners provide a name in the form of a diagnosis and this diagnosis often acts to shape the individual's identity. A client will often say "I am depressed" as opposed to "I suffer from depression." In this way, the client is identifying himself or herself as the diagnosis, rather than suffering from a disorder. Thus, both the naming ceremony and a diagnosis elicit a sense of identification with the name given to the individual.

Naming ceremonies and diagnosis do not overlap completely and it is these differences which may be of greatest concern. First of all, the names given in an Indigenous naming ceremony are honoured names (Thom, 2003) and are meant to enhance the person's sense of identity. Diagnoses, though they are meant to help the person and other understand what is going on, are often shameful names and can be deleterious to the person's sense of identity (Duran, 2006). Manson (2000) discusses how Aboriginal people who seek treatment fear being called a mental patient based on the diagnosis; this is reflective of the negative stigma attached to diagnostic labels as opposed to the positive associations with names given in a naming ceremony. A further difference is that names given in an Aboriginal naming ceremony are meant to reflect the holistic being (Duran, 2006) whereas a diagnosis is meant only to describe the part of the person afflicted by the disorder. This is problematic because an Aboriginal person who understands the diagnosis as a name being given to them will likely focus on the pathology of the name and may perceive it as describing their whole being rather than a specific part. This can make it more difficult for the person to cope because they believe they are the problem. 
In the Coast Salish people's tradition only one living person is given an honoured name (Thom, 2003) whereas a single diagnosis is used to describe numerous people. Diagnoses are not reflective of the unique characteristics of an individual whereas names given in a naming ceremony are designed to be unique. Furthermore, the names given in a naming ceremony are culturally relevant and based on the history and ancestry of the people (Thom, 2003). The diagnoses listed in the DSM-IV-TR are based on the history of the mainstream population, which is predominantly of white-European ancestry (Grob, 1991). As a result, the diagnoses given are not culturally based or appropriate for use with Aboriginal peoples.

The parallels and incongruence between diagnosis and a naming ceremony outlined above suggest that mental health practitioners must be careful when using diagnoses with Aboriginal people because of the underlying implications. A diagnosis given to someone from the dominant culture does not have connections to a traditional practice that is equivalent to a naming ceremony in Aboriginal cultures; consequently, when giving a diagnosis to an Aboriginal person it may carry more weight and meaning to the individual because the process resembles a naming ceremony (Duran, 2006). Conversely, the differences between a naming ceremony and being labelled with a diagnosis suggest that the practice of diagnosis is culturally inappropriate and potentially harmful (Duran, 2006). The limited literature on this topic suggests that many practitioners may not be aware of these parallels between providing a clinical diagnosis and an Aboriginal naming ceremony and as such it increases the potential for harm when working with Aboriginal clients, in the same way that any practice which is culturally inappropriate can be harmful (Duran, 2006; Stewart, 2008).

\section{Future Recommendations}

As Duran (2006) suggests, diagnosis is not completely without merit, it can serve a purpose in the healing process. Nevertheless, for diagnosis to be useful when working with Aboriginal people the diagnosis given must reflect Aboriginal worldviews, values and beliefs. The idea of having diagnosis match the values of Aboriginal people is daunting because of the vast heterogeneity of the people and cultures described by the term Aboriginal (Kirmayer et al., 2000; Stewart, 2008). Developing diagnosis to fit the unique aspects of all the different Aboriginal cultures may be impossible; however, a reasonable starting point would be to create diagnoses or a diagnostic system that respects values that are shared among Aboriginal cultures. Examples of such values are: the interrelatedness of all things; multiple realities and the lack of a single definitive truth; holistic conceptualization of people and health (Bopp \& Bopp, 2001;
Steinhauer, 2002). One of the primary experiences Aboriginal people share that needs to be recognized and included in the DSM-IV-TR is the concept of historical trauma. Both Duran (2006) and Evans-Campbell (2008) note this absence from the DSM-IV-TR and Duran suggests that by ignoring the impact of historical trauma that mental health professionals are unwittingly continuing to colonize clients.

The current process of review and revision of the DSM-IV emphasizes clinical evaluation studies which may not capture the intricacies of cultural influences (Csordas et al., 2008). At present the process of revision for the DSM-V is being conducted by 13 working groups of mental health professionals (APA, n.d.); yet, the working groups are relying on conference presentations and publications to formulate the new manual and there is very little opportunity for community consultation. The only opportunity for community consultation is a website where "the wider research, clinical, and consumer communities... could submit questions, comments, and research findings to be distributed to the relevant work groups" (APA, n.d.). Perhaps, the publishers of the DSM-IV-TR should reconsider the type of evidence they require for a particular disorder to be included in the manual if they want to better serve the diverse cultural populations which the manual attempts to address.

This article is not meant to argue that mental health professionals are maliciously attempting to colonize their clients; it is, however, arguing that mental health professionals must become aware of their impact on clients when they use tools and techniques that do not coincide with Aboriginal values and worldviews (Duran, 2006). Increased awareness may lead to the formulation of techniques and approaches that are congruent with Aboriginal values and worldviews. Both McCormick (1996) and Stewart (2008) discuss the implications for incorporating Aboriginal values and worldviews into counselling practice, which has the possibility to extend to other areas in the field of mental health. One way to increase awareness is through education; when mental health practitioners are educated, particularly at the post-secondary level, they should be taught about the colonial history of Aboriginal peoples and the deleterious impact of historical trauma. Moreover, practitioners should be encouraged to get to know the history of the Aboriginal people in the area in which they practice and familiarize themselves with the customs in order to better understand their clients (Duran, 2006). Progress has been made in this regard, as the Canadian government issued an official apology for the legacy of the residential school system (Offet-Gartner, 2008) and there is a growing body of research on historical trauma. For the practitioners who have completed their post-secondary education, information 
should be available through professional bodies and courses in continuing education. Literature and research will continue to provide an avenue for increasing awareness and providing educational opportunities.

The inclusion of the Outline for Cultural Formation in the DSM-IV is a useful starting point to encourage mental health professionals to think about the impacts of culture in the diagnostic process. Nevertheless, its placement in the Appendix section of the text does not give it the merit it deserves. As Kirmayer (1998) suggests, the Outline for Cultural Formation should be placed at the beginning of the text following the introduction to indicate its importance rather than being an add-on in the form of an appendix. So often suggestions for working with Aboriginal populations is an add-on to interventions based on mainstream values (Duran, 2006). In a field as important and strife with difficulties as mental health, professionals should be striving toward providing interventions that integrate Aboriginal worldviews and values, rather than positioning them as subservient to mainstream values by making any reference to them an add-on to existing systems. When working with Aboriginal clients, Aboriginal worldview and values, such as interconnectedness and respect for Elders, should provide the core to the interventions.

The changes suggested are not likely to happen overnight and the process to instate these changes is likely to be difficult and met with resistance. It is understandable that even mental health professionals who are reticent to using DSM-IV diagnoses may need to use diagnostic labels in order to help clients access resources and services or due to the requirements of the agency at which the mental health practitioner is employed. In the meantime, however, there are choices that mental health practitioners can make to reduce the negative impact that the diagnostic process is having on Aboriginal peoples. Duran (2006) suggests that a therapist can help a client re-name themselves or return to a name that was previously given to them and let go of the diagnosis as a defining part of his or her identity. This idea resonates with a tenant of Narrative therapy which suggests that the person is not the problem, the problem is the problem (Corey, 2005). This allows clients to dissociate their identity from the diagnosis and work with the problem/diagnosis is a new way (Duran, 2006). Duran also suggests using alternative terms for the diagnosis, such as "the spirit of sadness" when referring to depression ( $\mathrm{p} 80$ ). This places the diagnosis in more culturally relevant terms. Clinicians are encouraged to formulate strategies to work with Aboriginal clients that acknowledge and reflect the significance of the parallels between diagnosis and a naming ceremony. Development and application of these strategies is an important area to be addressed in future research.

\section{Summary and Conclusion}

There are numerous concerns about the mental health of Aboriginal peoples due to the high rates of mental health issues present among Aboriginal populations when compared to the Canadian population as a whole (Kirmayer et al., 2000; Kirmayer et al., 2003) There are existing barriers to service use which have been attributed to differences in Aboriginal and Western worldviews, while others suggest that historical trauma also impacts service use. Nonetheless, Aboriginal people who are accessing mental health services face a unique set of problems as a result of the diagnostic system in place.

The DSM-IV-TR is a clinical diagnosis tool that is based primarily on research within the dominant culture and the cultural components to the manual are presented in such a way that they seem to be add-ons rather than important concepts when dealing with Aboriginal peoples (Kirmayer, 1998). The lack of cultural relevance of the diagnoses provided in the DSM-IV has been a criticism, however, the newest inclusion of the "Outline for Cultural Formulation" represents movement in a more helpful direction.

Caution is warranted when using diagnostic labels with Aboriginal clients due to both the similarities and the differences between an Aboriginal naming ceremony and the process by which a person is given a diagnosis. Duran (2006) suggests using alternative terms and explanations for disorders that are culturally appropriate rather than relying solely on the DSM-IV diagnosis. At present, the DSM-IV is a tool based on in a Western model of healthcare; the need for culturally appropriate interventions and practices is becoming increasingly apparent. As the healthcare system evolves, so too will its tools; perhaps the upcoming revision, the DSM-V, will continue to incorporate culture into its structure and as Good (1996) points out, the inclusion of culture in a document such as the DSM continues to be a tremendous undertaking yet it has the potential to benefit many different groups of people, including Aboriginal peoples. It is also recommended that diagnostic labels, such as those provided in the DSM-IV-TR, be used primarily in documentations rather than with clients until more culturally relevant names and definitions can be found; nonetheless, when diagnostic labels are used clinicians must take the time to discuss these labels with clients to reduce the potentially negative impact and to address the significance of the label to the individual.

The changes suggested can occur at the level of individual mental health practitioners, by refraining from use of diagnostic labels and learning about Aboriginal history and culture, and also at a systemic level, such as changing the DSM-IV and the education of practitioners. Despite the difficulties that may be encountered when pursuing these changes the end result is the 


\section{First Peoples Child \& Family Review, Volume 5, Number 1, 2010}

provision of mental health care that is accessible, relevant and respectful to Aboriginal people.

\section{References}

American Psychiatric Association. (2009). Diagnostic and Statistical Manual. Retrieved March 29, 2009 from http://www.psych.org/MainMenu/ Research/DSMIV.aspx.

American Psychiatric Association. (2000). Diagnostic and Statistical Manual of Mental Disorders (4th ed.) Text Revision. Washington, DC: Author.

American Psychiatric Association. (1994). Diagnostic and Statistical Manual of Mental Disorders (4th ed.). Washington, DC: Author.

American Psychiatric Association. (n.d.). DSM-IV: The future manual. Retrieved from http://www.psych.org/dsmvasp.

Beck, A. T. (1991). Cognitive therapy: A 30-year retrospective. American Psychologist, 46 (4), 368-375.

Bopp, M \& Bopp, J. (2001). Recreating the world: A practical guide to building sustainable communities. Lethbridge, AB: Four Worlds Press.

Castellano, M. B. (1999). Updating traditions of Aboriginal knowledge. In B. L. Hall, G. J. S. Dei, \& D. G. Rosenberg (Eds.) Indigenous Knowledges in Global Contexts, pp. 21-36. Toronto, ON: University of Toronto Press.

Corey, G. (2005). Theory and practice of counseling and psychotherapy. California: Brooks/Cole.

Csordas, T.J., Storck, M. J., \& Strauss, M. (2008). Diagnosis and distress in Navajo healing. The Journal of Nervous and Mental Disease, 196 (8), 585-596.

Dziegielewski, S. F. (2002). DSM-IV-TR in Action. New York: John Wiley \& Sons.

Duran, E., Firehammer, J. \& Gonzalez, J. (2008). Liberation Psychology as a path toward healing cultural soul wounds. Journal of Counselling and Development, 86, 288-295.

Duran, E. (2006). Healing the Soul Wound: Counselling with American Indians and other Native Peoples. New York: Teachers College, Columbia University.

Evans-Campbell, T. (2008). Historical trauma in American Indian/Alaska Natives: A multilevel framework for exploring impacts on Individuals, families and communities. Journal of Interpersonal Violence, 23(3), 316-328.

Good, B. J. (1996). Culture and DSM-IV: Diagnosis, knowledge and power. Culture, Medicine \& Psychiatry, 20, 127-132.

Greden, J. F. (2003). Physical symptoms of depression: Unmet needs. Journal of Clinical Psychiatry, 64, 5-11.

Grob, G. N. (1991). The origins of the DSM-I: A study in appearance and reality. The American Journal of Psychiatry, 148 (4), 421-431.

Kirmayer, L. J. (2004). The cultural diversity of healing: meaning, metaphor and mechanism. British Medical Bulletin, 69, 33-48.

Kirmayer, L. J. (1998). The fate of culture in the DSM. Transcultural Psychiatry, 35(3), 339-342

Kirmayer, L. J., Brass, G. M., \& Tait, C. L. (2000). The mental health of aboriginal peoples: Transformations of identity and community. Canadian Journal of Psychiatry, 45 (7), 607-616.
Kirmayer, L. J., Simpson, C., \& Cargo, M. (2003). Healing traditions: culture, community and mental health promotion with Canadian Aboriginal peoples. Australasian Psychiatry, 11 (Suppl. 1), 15-23.

Leahy, R. L. \& Holland, S. J. (2000). Treatment plans and interventions for depression and anxiety disorders. New York: Guilford Press.

Manson, S. M. (2000). Mental health services for American Indians and Alaska Natives: need use and barriers to effective care. The Canadian Journal of Psychiatry, 45, 617-626.

Markowitz, F. E. (1998). The effects of stigma on the psychological well-being and life satisfaction of persons with mental illness. Journal of Health and Social Behaviour, 39 (4), 335-347.

McCabe, G. (2008). Mind, body, emotions and spirit: reaching to the ancestors for healing. Counselling Psychology Quarterly, 21(2), $143-152$.

McCormick, R. (1996). Culturally appropriate means and ends of counselling as described by the First Nations of British Columbia. Journal for the Advancement of Counselling, 18, 163-172.

McIntyre, M. P.J. (1996). Counselling and Native healing. Asian Journal of Counselling, 5(1), 87-100.

Offet-Gartner, K. (2008).Sharing the story: Education as the key to unlocking the door of career possibilities with First Nations women. Available from Dissertation Abstracts International. Publication No. AAT NR44363.

Schissel, B. \& Wotherspoon, T. (2003). The Legacy of School for Aboriginal People: Education, Oppression and Emancipation. Don Mills, ON: Oxford University Press.

Steinhauer, E. (2002). Thoughts on an Indigenous research methodology. Canadian Journal of Native Education, 26 (2), 69-81.

Stewart, S. L. (2007). Indigenous helping and healing in counsellor training. Center for Native Policy and Research Monitor, 2 (1), 35-63.

Stewart, S. L. (2008) Promoting Indigenous mental health: Cultural perspectives on healing from Native counsellors in Canada. International Journal of Health Promotion and Education, 46, 2, 49-56.

Thom, B. (2003, September). Intangible Property within Coast Salish First Nations Communities, British Columbia. Paper presented at WIPO North American Workshop on Intellectual Property and Traditional Knowledge. Retrieved from http://home.istar.ca/ bthom/paper-wipo. pdf.

Van Den Tillaart, S., Kurtz, D. \& Cash, P. (2009). Powerlessness, marginalized identity and silencing of health concerns: Voiced realities of women living with a mental health diagnosis. International Journal of Mental Health Nursing, 8 (3), 153-163.

Vicary, D. \& Bishop, B. (2005). Western psychotherapeutic practice: Engaging Aboriginal people in culturally appropriate and respectful ways. Australian Psychologist, 40(1), 8-19.

Whitbeck, L., Adams, G., Hoyt, D., \& Chen, X. (2004). Conceptualizing and measuring historical trauma among American Indian people. American Journal of Community Psychology, 33 (3), 119-130.

Wilson, K. \& Rosenberg, M. W. (2002). Exploring the determinants of health for First Nations peoples in Canada: can existing frameworks accommodate traditional activities? Social Sciences \& Medicine, 55, 2017-2031 